\title{
Complex Relationship between COVID-19 and BCG Vaccination Policy
}

\author{
Jogender Kumar ${ }^{1}$, Arushi Yadav², and Jitendra Meena ${ }^{1}$ \\ ${ }^{1}$ PGIMER \\ ${ }^{2}$ Government Medical College and Hospital Department of Community Medicine
}

July 8, 2020

Dear Editor,

We read the article on possible effects of BCG vaccination policy on COVID-19 spread by Ozdemir et al. with interest and found it very thoughtful. ${ }^{1}$ In early stages of the COVID-19 pandemic, the countries without universal vaccination policies (Italy, United States) were more severely affected as compared to those with universal vaccination policies (India, Brazil). ${ }^{2}$ Based on these epidemiological observations it was hypothesized that the non-specific effects of BCG vaccine might be protective against SARS-CoV-2 infection and many randomized trials are testing it. ${ }^{2-4}$ However, we have a few concerns over these epidemiological associations.

Firstly, most authors including Ozdemir et al divided the countries into two groups according to the presence or absence of "recent routine childhood BCG vaccination policy". The countries having universal BCG coverage above $90 \%$ previously and stopped routine vaccination practice in the last one or two decades only (Czechia, Finland, France, Ireland, Norway, Slovakia, United Kingdom) were classified into the countries with "no BCG-vaccination policy". On the contrary, some of the countries with routine BCG vaccination policy but poor coverage (Nigeria, Somalia, South Sudan) were included in the category of countries following "routine BCG-vaccination policy". Do the authors mean to confer that the protection offered by BCG (by its non-specific effects on immunity) is sustained for one to two decades only, and the actual immunization coverage of a country does not play a role? In that scenario, BCG vaccination shall be protective for children only (the least-affected age-group with COVID-19), whereas the adults and geriatric population (most-affected) will not be benefitted by it. Such classification (according to recent BCG vaccination status) completely ignores the previous vaccination status, actual BCG-vaccination coverage, and generalizes the results across all age-groups and leads to false conclusions. Therefore, an age-specific comparison of COVID19 and BCG immunization coverage might be more meaningful. ${ }^{5}$

Secondly, the authors did not adjust for any confounding factors like the number of tests done at that time, population demographics, co-morbidities, health infrastructure, reporting bias, etc. that can significantly alter the results. ${ }^{3}$ A recent study observed a positive correlation (opposite to what we think) between the COVID-19 related parameters (cases, death-rate, and case-fatality rate) and BCG vaccination coverage of various countries across the span of four decades. However, with adjustment of confounders, there was no correlation between COVID-19 and BCG coverage. ${ }^{6}$

Thirdly, at the time of analysis (April 16, 2020) the pandemic was limited to the northern hemisphere which now has rapidly evolved. For now, four (India, Brazil, Russia, Peru) out of the five most affected countries have routine BCG vaccination policy with more than $90 \%$ coverage. ${ }^{5,6}$ Therefore, the analysis favouring BCG vaccination in the initial stages of the pandemic was too early to predict and was affected by the limited spread of COVID-19. To test this hypothesis, we analyzed the correlation between BCG coverage (2010- 
2018) and COVID-19 related parameters (Cases per million, and deaths per million) of various countries at two different time-points (March 01, 2020, and June 29, 2020) using various datasets. ${ }^{7,8}$ We observed a weak but significant positive correlation (spearmen $\mathrm{rho}=+0.2-0.4, \mathrm{p}<0.05$ ) between the $\mathrm{BCG}$ vaccination coverage and COVID-19 cases and deaths (as of March 01, 2020). However, there was no correlation on June 29, 2020 (Table 1). Moreover, a recent study observed that the BCG vaccination in childhood was not protective against SARS-CoV-2 infection. ${ }^{4,5}$

Based upon the above, we conclude that as of now there is no correlation between recent BCG vaccination coverage of a country and COVID-19. As the protective efficacy may not last beyond childhood, we should not equate the childhood vaccination policy of a country with the recent vaccination in ongoing trials. Until we have the results of ongoing randomized clinical trials, routine use of BCG vaccine in COVID-19 management should be discouraged and restricted to research purpose only. ${ }^{2}$

\section{References}

1. Ozdemir C, Kucuksezer UC, Tamay ZU. Is BCG vaccination affecting the spread and severity of COVID-19? Allergy. Published online May 12, 2020:all.14344. doi:10.1111/all.14344

2. Patella V, Florio G, Raffaele B, Delfino G. Could Anti-Tubercular Vaccination Protect Against Covid19 Infection? Allergy. Published online Jun 23, 2020;all.14443. doi: 10.1111/all.14443

3. Kumar J, Meena J. Demystifying BCG Vaccine and COVID-19 Relationship. Indian Pediatr. 2020;57(6):588-589.

4. Riggioni C, Comberiati P, Giovannini M, Agache I, Akdis M, Alves-Correia M, et al. A compendium answering 150 questions on COVID-19 and SARS-CoV-2. Allergy. Published online June 14, 2020;all.14449. doi: 10.1111/all.14449

5. Hamiel U, Kozer E, Youngster I. SARS-CoV-2 Rates in BCG-Vaccinated and Unvaccinated Young Adults. JAMA. Published online May 13, 2020. doi:10.1001/jama.2020.8189

6. Meena J, Yadav A, Kumar J. BCG Vaccination Policy and Protection Against COVID-19. Indian J Pediatr. Published online June 9, 2020. doi:10.1007/s12098-020-03371-3

7. World Health Organization. Coronavirus Disease (COVID-2019) Situation Reports. Available at: https://www.who.int/emergencies/diseases/novel-coronavirus-2019/situation-reports. Accessed 02 July 2020 .

8. World Health Organization. BCG Immunization Coverage Estimates by Country. Global Health Observatory Data Repository. Available at: https://apps.who.int/gho/data/view.main.80500?lang=en. Accessed 02 July 2020.

Table 1. Correlation between BCG vaccine coverage (2010-2018) of countries with the COVID-19 Cases and Deaths

\begin{tabular}{llllllll}
\hline Time Points & COVID-19 Parameters (per million population) & Years & Years & Years & Years & Years & Years \\
\hline \multirow{3}{*}{ June 29, 2020 } & Total Cases & $\mathbf{2 0 1 8}$ & $\mathbf{2 0 1 7}$ & $\mathbf{2 0 1 6}$ & $\mathbf{2 0 1 5}$ & $\mathbf{2 0 1 4}$ & $\mathbf{2 0 1 3}$ \\
& Deaths & 0.12 & 0.10 & 0.06 & 0.09 & 0.13 & 0.12 \\
& No. of Countries (n) & 0.01 & -0.03 & -0.05 & -0.19 & 0.03 & 0.04 \\
March 01, 2020 & Total Cases & 140 & 140 & 141 & 142 & 142 & 142 \\
& Deaths & $0.42^{*}$ & $0.38^{*}$ & $0.35^{*}$ & $0.34^{*}$ & $0.34^{*}$ & $0.37^{*}$ \\
& No. of Countries (n) & $0.21^{* *}$ & $0.18^{* *}$ & $0.15^{* *}$ & $0.17^{* *}$ & $0.20^{* *}$ & $0.22^{* *}$ \\
& & 133 & 133 & 134 & 135 & 135 & 135 \\
\hline
\end{tabular}

*Correlation is significant at p-0.01 level

${ }^{* *}$ Correlation is significant at p- 0.05 level

Hosted file

blinded manuscript_05.07.docx available at https://authorea.com/users/340032/articles/467861- 
complex-relationship-between-covid-19-and-bcg-vaccination-policy 